\title{
Correction to: Analysis of plasma multiplex cytokines and increased level of IL-10 and IL-1Ra cytokines in febrile seizures
}

Kyungmin Kim', Byung Ok Kwak', Aram Kwon', Jongseok Ha', Soo-Jin Kim², Sun Whan Bae², Jae Sung Son², Soo-Nyung Kim ${ }^{3}$ and Ran Lee $2,4^{*}$

\section{Correction}

After publication of the article [1], it has been brought to our attention that several of the authors' names were formatted incorrectly in the original version of the article. The corrections are listed below -

"Byungok Kwak" should be "Byung Ok Kwak"

"Soojin Kim" should be "Soo-Jin Kim"

"Sunwhan Bae" should be "Sun Whan Bae"

"Jaesung Son" should be "Jae Sung Son"

"Soonyung Kim" should be "Soo-Nyung Kim"

The original version of the article has now been revised.

\begin{abstract}
Author details
'Department of Pediatrics, Konkuk University Medical Center, Seoul, South Korea. ${ }^{2}$ Department of Pediatrics, Konkuk University Medical Center, Konkuk University School of Medicine, 120-1 Neungdong-ro (Hwayang-dong), Gwangjin-gu, Seoul 05030, South Korea. ${ }^{3}$ Department of Obstetrics and Gynecology, Konkuk University Medical Center, Konkuk University School of Medicine, Seoul, South Korea. ${ }^{4}$ International Healthcare Research Institute,

Konkuk University, Seoul, South Korea.
\end{abstract}

Published online: 17 November 2017

\section{Reference}

1. Kim K, Kwak BO, Kwon A, Ha J, Kim S, Bae SW, et al. Analysis of plasma multiplex cytokines and increased level of IL-10 and IL-1Ra cytokines in febrile seizures. J Neuroinflammation. 2017;14:1. https://doi.org/10.1186/ s12974-017-0974-7.

\footnotetext{
* Correspondence: 20050069@kuh.ac.kr

The online version of the original article can be found under doi:10.1186/ s12974-017-0986-3

${ }^{2}$ Department of Pediatrics, Konkuk University Medical Center, Konkuk University School of Medicine, 120-1 Neungdong-ro (Hwayang-dong), Gwangjin-gu, Seoul 05030, South Korea

${ }^{4}$ International Healthcare Research Institute, Konkuk University, Seoul, South Korea
} 